\title{
The prognostic value of combination of CD90 and OCT4 for hepatocellular carcinoma after curative resection
}

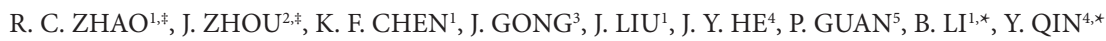 \\ ${ }^{1}$ Department of Liver Surgery and Liver Transplantation Center, West China Hospital, Sichuan University, Chengdu 610041, China; ${ }^{2}$ Department \\ of Medical Oncology, Cancer Center, State Key Laboratory of Biotherapy, West China Hospital, Sichuan University, Chengdu 610041, China; \\ ${ }^{3}$ Department of Pathology, West China Hospital, Sichuan University, Chengdu 610041, China; ${ }^{4}$ Department of Biochemistry and Molecular Biol- \\ ogy, West China School of Preclinical and Forensic Medicine, Sichuan University, Chengdu 610041, China; Department of Forensic Pathology, \\ West China School of Preclinical and Forensic Medicine, Sichuan University, Chengdu 610041, China
}

*Correspondence: cdlibo168@hotmail.com,qin_1@sina.com

${ }^{*}$ Contributed equally to this work.

Received August 1, 2015 / Accepted September 21, 2015

\begin{abstract}
CD90 has been identified as a candidate marker of cancer stem cells (CSCs) for HCC, whereas it also has been considered as a marker for tumor-associated fibroblasts (CAFs). OCT4, as a key transcription factor required to maintain pluripotency of human embryonic stem cell and cancer cells, has been characterized to be involved in malignant transformation and tumorigenesis of various cancers. This study aimed to examine expression patterns of CD90 in HCC and investigate whether combination of both CD90 and OCT4 could provide a more powerful predictor for prognosis of HCC than either one alone.

CD90 and OCT4 were examined by immunohistochemistry. The relationship between CD90/OCT4 expression and clinicopathologic characteristics was analyzed. The correlation between CD90/OCT4 expression and overall survival and disease-free survival was determined with Kaplan-Meier analysis.

CD90 was found mainly expressed in tumor-associated CAFs and OCT4 was mainly expressed in tumor cells. The expression of CD90 and OCT4 in HCC was significantly higher than in adjacent non-tumor and normal liver tissues. CD90 expression was correlated with pathological grade, satellite lesion, PVTT and recurrence. OCT4 expression was correlated with pathological grade, tumor size and recurrence. Data demonstrated no correlation between CD90 and OCT4. High expression of CD90 or OCT4 predicts a poor prognosis. Furthermore, combination of both CD90 and OCT4 provides a more sensitive predictor for prognosis of HCC than either marker alone.

CD90 and OCT4 are both independent and reliable biomarker for predicting prognosis of HCC patients after hepatic resection. Our results indicated the accuracy of prediction can be enhanced by their combination.
\end{abstract}

Key words: CD90, OCT4, HCC, prognosis, immunohistochemistry

Hepatocellular carcinoma (HCC) is one of the most aggressive malignancies worldwide, especially in China, East Asia and South Africa [1]. The main risk factors associated with HCC are chronic hepatitis B or hepatitis C, aflatoxin B and alcoholism [2-5]. Despite great improvement in the curative treatments, such as surgical resection, radiofrequency ablation and liver transplantation, the long-time survival after HCC resection still remains unsatisfactory, because of a high incidence of recurrence [6]. So it is very important and urgent to find an effective biomarker to identify patients with a high risk of recurrences, and provide appropriate therapy to reduce the risk.
Recently, the concept of tumor microenvironment (TME) had been proposed and extensively accepted that tumor cells and tumor-associated stroma cells interact with each other and influence disease initiation, progression and prognosis together [7]. In the TME, cancer-associated fibroblasts (CAFs), a main kind of tumor-associated stroma cells, are distinct from normal fibroblasts and are present in aberrantly high numbers. CAFs play a crucial role in tumorigenesis and tumor metastasis by providing a major source of secreted growth factors [8].

CD90 (also known as Thy-1) is a $25-37 \mathrm{kDa}$ cell surface glycoprotein. In humans, it is mainly expressed on several types of cells including: fibroblasts, blood stem cells and 
activated microvascular endothelial cells (ECs) $[9,10]$. Nevertheless, high level of CD90 expression has also been observed in CAFs [11-13]. Sukowati et al [14] found that CAFs isolated from human HCC tissues were positive for CD90. In addition, Chen et al' study [15] identified CD90 as one of markers of CAFs and demonstrated that $\mathrm{CD} 90^{+} \mathrm{CAFs}$ promoted stem-like properties in lung cancer cells. However, the prognostic value of CD90 as the biomarker of CAFs is not well-known.

OCT4 (also known as POU5F1) is one of embryonic stem cell markers. OCT4 plays an important role in the maintenance of the stemness properties $[16,17]$. Normally, expression of OCT4 is confined to pluripotent cells in the developing embryo $[18,19]$. Recently, OCT4 expression had been observed in cancer cells of some malignancies, such as breast and prostate cancer, bladder cancer and HCC [20-23]. Latest studies demonstrated that OCT4 plays crucial roles in the malignant behavior of embryonic stem cells (ESCs) [24]. What's more, OCT4 inactivation leaded to regression of the malignancy, indicating significance of OCT4 for maintaining the malignant phenotype and determining the oncogenic fate of ESCs [25]. However, relatively little is known about the prognostic value of OCT4.

It is therefore of interest to investigate CD90 and OCT4 as potential and reliable biomarkers for HCC patients after curative resection. Though there have been studies reporting prognostic value of OCT4 for HCC [26], testing combination of CD90 and OCT4 for prediction of prognosis has not been previously investigated. The prognostic accuracy of testing can be enhanced by combination of them, because effects of tumor cells and stroma cells were both taken into consideration by the combination.

\section{Methods}

Ethics. This study was approved by the medical ethics committee of the West China hospital, Sichuan University. All participating subjects voluntarily offered written informed consent. All clinical investigations were conducted in accordance with the principles expressed in the Declaration of Helsinki.

Patients and tissue samples. Paraffin-embedded liver specimens used in this study were obtained from the West China hospital, Chengdu, China. HCC tissue samples $(\mathrm{n}=86)$ and corresponding adjacent non-tumor tissues $(n=86)$ were obtained from patients who underwent curative resection form April 2009 to March 2011. The diagnoses of these HCC samples were verified by senior pathologists. None of the patients had received prior radiotherapy or chemotherapy treatment when the tissue samples were collected. Complete pathological and clinical data were all collected and described in Table 2. Cirrhosis samples $(n=22)$ and hepatitis samples $(\mathrm{n}=11)$ were collected from individuals without cancers who went though hepatic resection or hepatic puncture biopsy. In addition, 10 normal liver tissues were obtained from adult patients who underwent hepatectomy for benign conditions such as hemangioma. All samples were obtained with previous written consent.

Follow-up. Post-operative patients were followed up once a month during the first half year, and then every 3 months afterwards. Serum AFP level and abdominal ultrasonography were performed each time during the routine review. Computed tomography (CT) was conducted every 3 to 6 months after operation. Survival status of all patients was obtained from patient records or follow-up telephone calls by the endpoint of April 2015. Date of death for each patient was verified by local civil affairs department. Survival time was calculated from the date of operation to the date of death or the last follow-up. The median follow-up period was 31.5 months (range 3 to 72 months). Recurrence was confirmed on the basis of positron emission tomography -CT (PET-CT), or the combination of increased serum AFP levels and evidences from ultrasonography, CT, magnetic resonance imaging (MRI), according to the latest guidelines released. Increase in serum AFP alone was not considered as evidence of recurrence.

Immunohistochemical (IHC) staining. The paraffinembedded tissues were cut into 4 -mm-thick sections for IHC staining using a standard two-step method. After the slides were deparaffinized and rehydrated, antigen retrieval was achieved in $10 \mathrm{mM}$ sodium citrate buffer ( $\mathrm{pH}$ 6.0) for $30 \mathrm{~min}$ at $98^{\circ} \mathrm{C}$. Then, slides were treated with $3 \%$ hydrogen peroxide $20 \mathrm{~min}$ for the inactivation of endogenous peroxidase.

After blocking for $30 \mathrm{~min}$, sections were incubated with mouse anti-CD90 (Abcam, ab181469, at 1:400 dilution) or rabbit anti-OCT4 (Abcam, ab19857, at 1:150 dilution) primary antibodies overnight at $4^{\circ} \mathrm{C}$. After washing, slides were then incubated with HRP-labeled secondary antibody for $40 \mathrm{~min}$ at $37^{\circ} \mathrm{C} \cdot 3,3^{\prime}$-Diaminobenzidine tetrahydrochloride (DAB) was used as the suitable substrate-chromogen and Mayer's

Table 1. The expression of CD90 and OCT4 in different types of liver diseases

\begin{tabular}{lccccc}
\hline \multirow{2}{*}{ Group } & N & \multicolumn{2}{c}{ Expression of CD90 } & \multicolumn{2}{c}{ Expression of OCT4 } \\
\cline { 3 - 6 } & & High & Low & High & Low \\
\hline Normal liver & 10 & $2(20.0 \%)$ & $8(80.0 \%)$ & $4(0 \%)$ & $10(100.0 \%)$ \\
Hepatitis & 11 & $6(54.5 \%)$ & $5(45.6 \%)$ & $4(36.4 \%)$ & $7(63.6 \%)$ \\
Cirrhosis & 22 & $19(86.4 \%)$ & $3(13.6 \%)$ & $6(27.3 \%)$ & $16(72.7 \%)$ \\
Adjacent non-tumor & 86 & $41(47.7 \%)$ & $45(52.3 \%)$ & $30(34.9 \%)$ & $56(65.1 \%)$ \\
HCC & 86 & $58(67.4 \%)$ & $26(32.6 \%)$ & $52(60.5 \%)$ & $34(39.5 \%)$ \\
\hline
\end{tabular}


hematoxylin was used for nuclear counterstaining. Negative controls were treated the same way, replacing the primary antibody by mouse or rabbit IgG..

Evaluation of staining. Three random fields $(\times 400)$ were examined and photographed with a Leica optical microscope. Two pathologists (Gong and Guan) who were blinded to the clinical data examined the slides independently. Immunohistochemical staining was evaluated by using the immunoreactivity score (IRS) as previously described [27]. The details were as follows: The staining intensity was scored as 0 (negative), 1 (weak), 2 (moderate), and 3 (strong). The extent of staining was scored as $0(0 \%), 1(<10 \%), 2(10 \%-50 \%)$,
$3(51 \%-80 \%)$, and $4(>80 \%)$, according to the percentages of the positive staining areas in relation to the entire carcinoma. The IRS (0-12) was then calculated by multiplying the score values. For the purpose of staining evaluation, sections were graded as high expression (total score $\geq 4$ ) and low expression (total score $<4$ ).

Statistical analysis. Statistical analyses were performed with the SPSS version 19.0 software (Chicago, IL, USA) and GraphPad Prism version 5.0 (La Jolla, CA). The MannWhitney $U$ test was employed to compare expression level of CD90 and OCT4 among different types of liver diseases. Chi-square test or Fisher's exact test was used to assess dif-

Table 2. Correlation between clinicalpathological characteristics and expression of CD90 and OCT4 in HCC patients

\begin{tabular}{|c|c|c|c|c|c|c|c|}
\hline \multirow{2}{*}{ Variables } & \multirow{2}{*}{ Case } & \multicolumn{2}{|c|}{ CD90 expression } & \multirow{2}{*}{ P value } & \multicolumn{2}{|c|}{ OCT4 expression } & \multirow{2}{*}{$P$ value } \\
\hline & & High & Low & & High & Low & \\
\hline Gender & & 58 & 28 & & 52 & 34 & \\
\hline Male & 67 & 46 & 21 & 0.652 & 42 & 25 & 0.429 \\
\hline Female & 19 & 12 & 7 & & 10 & 9 & \\
\hline \multicolumn{8}{|l|}{ Age (years) } \\
\hline$<60$ & 69 & 46 & 23 & 0.757 & 42 & 27 & 0.518 \\
\hline$\geq 60$ & 17 & 12 & 5 & & 10 & 7 & \\
\hline \multicolumn{8}{|l|}{ HBsAg } \\
\hline Negative & 13 & 7 & 6 & 0.337 & 6 & 7 & 0.252 \\
\hline Positive & 73 & 51 & 22 & & 46 & 27 & \\
\hline \multicolumn{8}{|l|}{$\operatorname{AFP}(\mathrm{ng} / \mathrm{ml})$} \\
\hline$<400$ & 42 & 31 & 11 & 0.218 & 21 & 21 & 0.077 \\
\hline$\geq 400$ & 44 & 27 & 17 & & 31 & 13 & \\
\hline \multicolumn{8}{|l|}{ Cirrhosis } \\
\hline Yes & 45 & 33 & 12 & 0.222 & 28 & 17 & 0.727 \\
\hline No & 41 & 25 & 16 & & 24 & 17 & \\
\hline \multicolumn{8}{|c|}{ Tumor Size $(\mathrm{cm})$} \\
\hline$<5$ & 39 & 26 & 13 & 0.889 & 19 & 20 & $0.042^{*}$ \\
\hline$\geq 5$ & 47 & 32 & 15 & & 33 & 14 & \\
\hline \multicolumn{8}{|c|}{ Pathological grade } \\
\hline WD & 12 & 5 & 7 & $0.030^{*}$ & 3 & 9 & $0.008^{*}$ \\
\hline MD & 57 & 38 & 19 & & 35 & 22 & \\
\hline $\mathrm{PD}$ & 17 & 15 & 2 & & 14 & 3 & \\
\hline \multicolumn{8}{|c|}{ Tumor encapsulation } \\
\hline Complete & 48 & 32 & 16 & 0.873 & 28 & 20 & 0.650 \\
\hline Incomplete & 38 & 26 & 12 & & 24 & 14 & \\
\hline \multicolumn{8}{|l|}{ Satellite lesion } \\
\hline Yes & 18 & 16 & 2 & $0.029^{*}$ & 13 & 5 & 0.251 \\
\hline No & 68 & 42 & 26 & & 39 & 29 & \\
\hline \multicolumn{8}{|l|}{ PVTT } \\
\hline Yes & 30 & 25 & 5 & $0.021^{*}$ & 19 & 11 & 0.690 \\
\hline No & 56 & 33 & 23 & & 33 & 23 & \\
\hline \multicolumn{8}{|l|}{ Recurrence } \\
\hline Yes & 59 & 45 & 14 & $0.010^{*}$ & 43 & 16 & $<0.001^{*}$ \\
\hline No & 27 & 13 & 14 & & 9 & 18 & \\
\hline
\end{tabular}

* Statistically significant $(\mathrm{P}<0.05)$

HBsAg, hepatitis B surface antigen; AFP, Alpha-fetoprotein; PD, poorly differentiated; MD, moderately differentiated; WD, well differentiated; PVTT, portal vein tumor thrombi; 
ferences between categorical variables. Spearman correlation test and Chi-square test were performed to evaluate the correlation between CD90 and OCT4 expression. Survival analyses were determined using the Kaplan-Meier method with GraphPad Prism v 5.0. Log-rank test was applied to compare survival of patients between subgroups. Univariable analysis was firstly used to determine prognostic factors for patients. Then variables with statistical significance from the univariable analysis were entered in the multivariable analysis, performed with the Cox-proportional hazards model with forward stepwise selection. $\mathrm{P}<0.05$ was considered statistically significant.

A
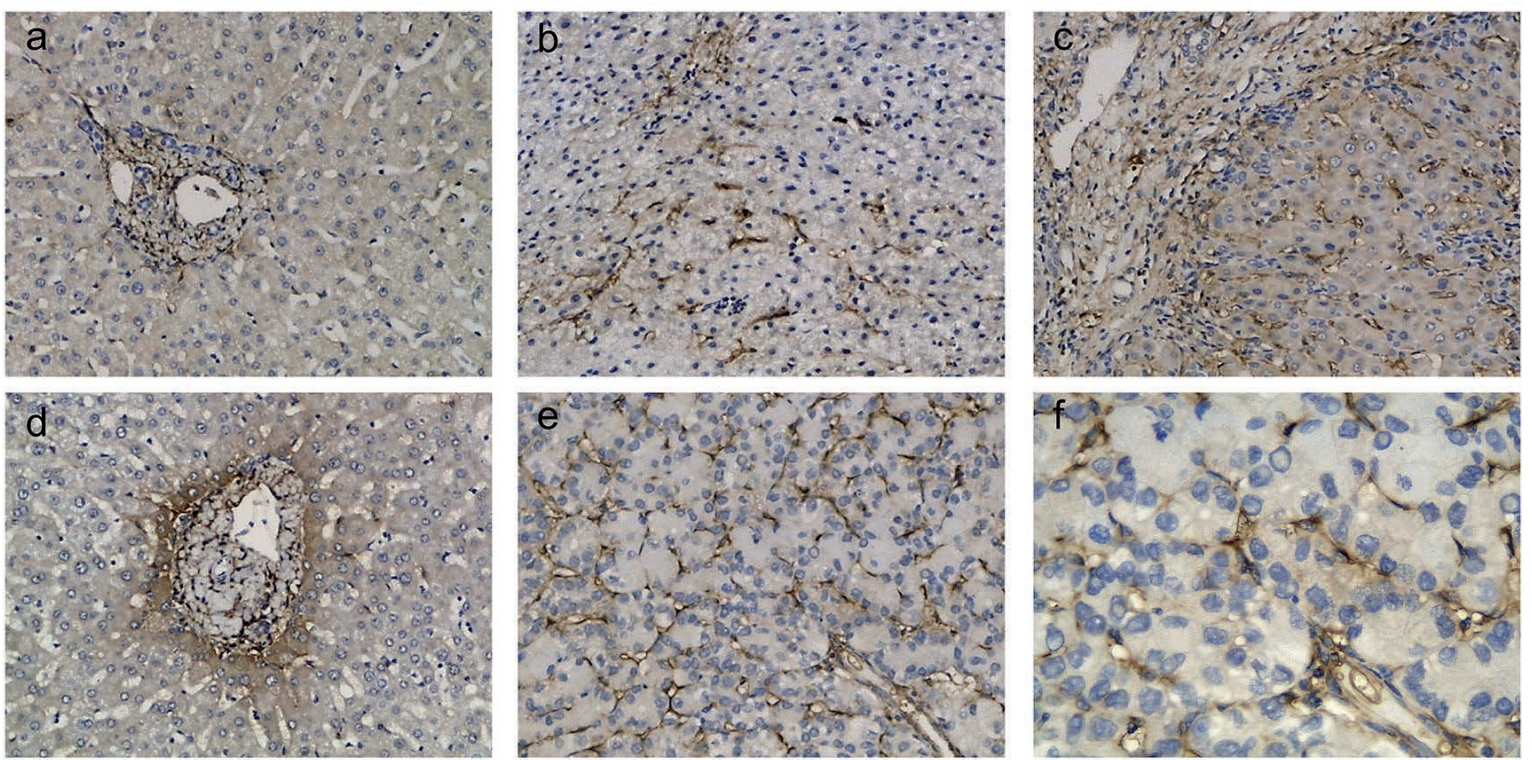

B

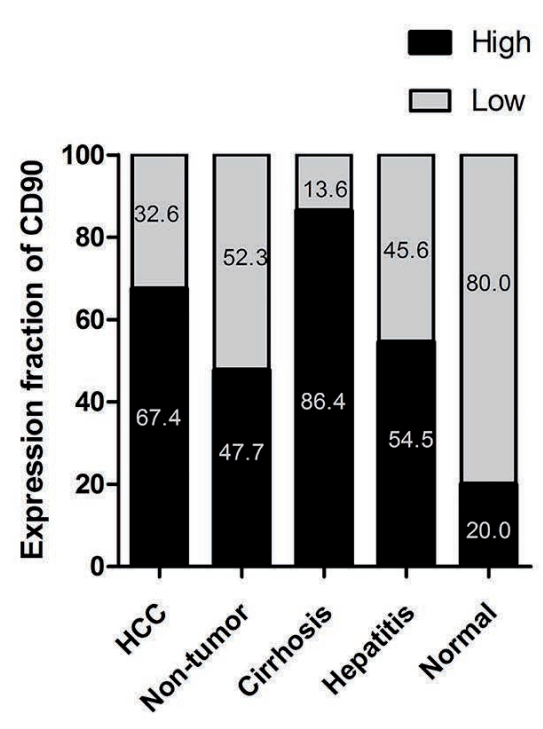

C
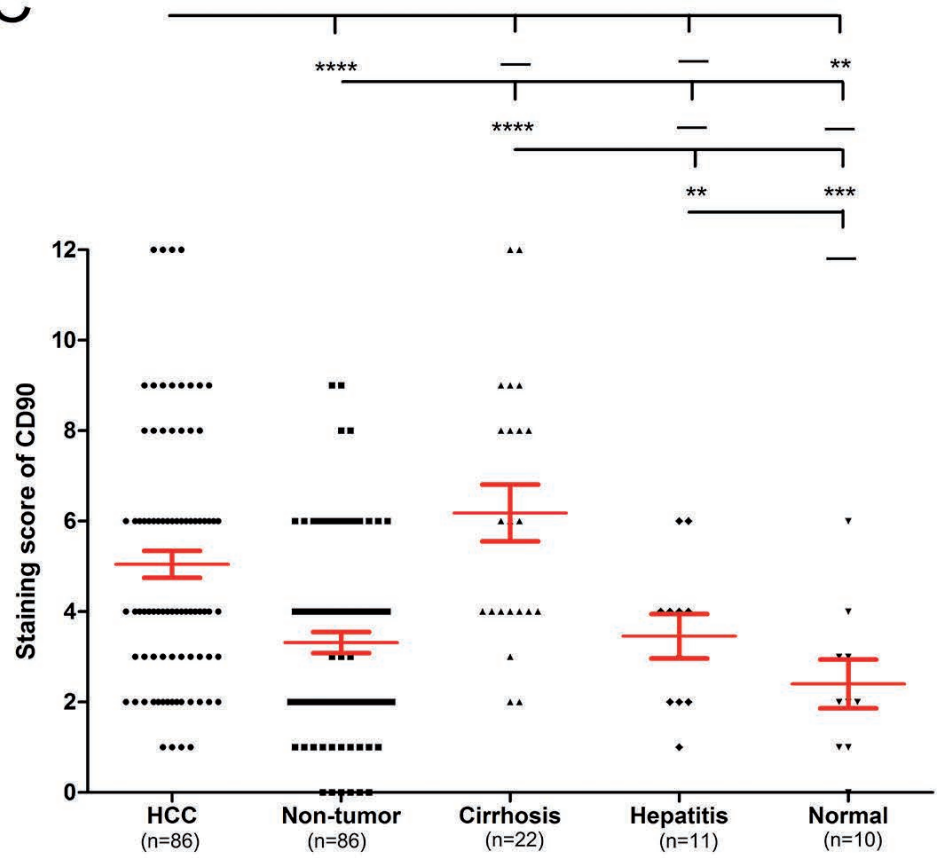

Figure 1. CD90 expression in different types of liver diseases. (A) Immunohistochemical staining in normal liver (a), hepatitis (b), cirrhosis (c), adjacent non-tumor (d), and HCC (e, f). Original magnification: $100 \times(\mathrm{a}-\mathrm{e})$ and $400 \times(\mathrm{f})$. (B) Expression fraction of CD90. (C) Comparison of staining score of CD90 expression among different types of liver diseases. 


\section{Results}

Expression patterns of CD90 in different types of liver diseases. We examined expression of CD90 in 86 pairs of HCC and adjacent non-tumor tissues, 22 cirrhosis, 11 hepatitis and 10 normal liver tissues using immunohistochemistry staining. In normal livers, CD90 only showed weak and sparse staining in the portal tract area. Only $20 \%$ normal liver tis- sues expressed high level of CD90 (2/10). While in chronic hepatitis, CD90 expression was observed in the activated fibroblasts, with a higher rate of $54.5 \%(6 / 11)$. Whereas in HCC tissues, CD90 was abundantly expressed in activated stroma cells, including CAFs and vascular endothelial cells, and the CD90 high expression rate increased to $67.4 \%(58 / 86)$. Besides, staining of adjacent non-tumor tissues $(47.7 \%, 41 / 86)$ showed CD90 expression extended to hepatocytes around the

A
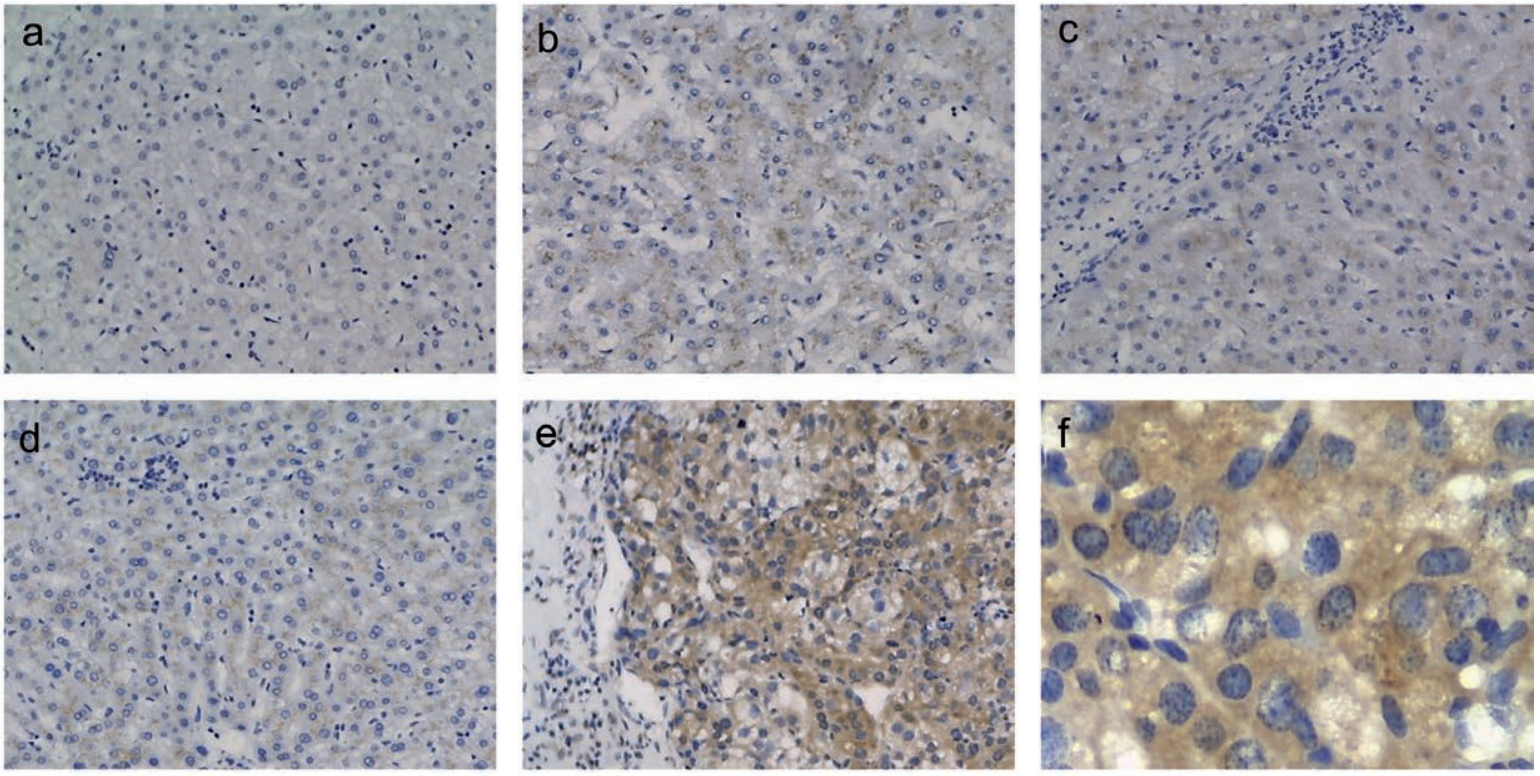

B

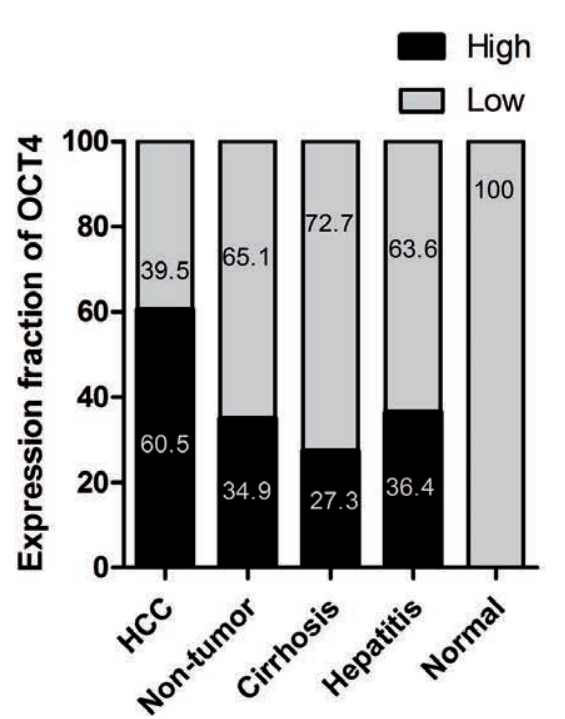

C

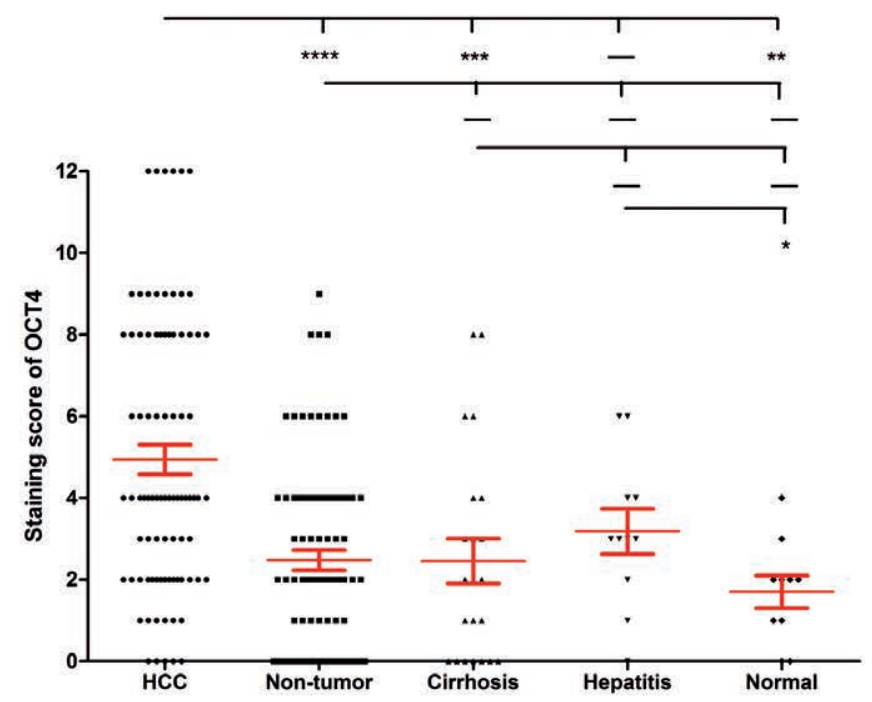

Figure 2. OCT4 expression in different types of liver diseases. (A) Immunohistochemical staining in normal liver (a), hepatitis (b), cirrhosis (c), adjacent non-tumor (d), and HCC (e, f). Original magnification: $100 \times(\mathrm{a}-\mathrm{e})$ and $400 \times(\mathrm{f})$. (B) Expression fraction of OCT4. (C) Comparison of staining score of OCT4 expression among different types of liver diseases. 
portal tract area, except for stroma cells. Notably, in cirrhosis, CD90 was highly over expressed. 86.4\% (19/22) of cirrhosis specimens presented high expression of CD90, with strong staining in fibroblasts surrounding the regeneration nodules (Table 1, Fig. 1A, B).

Significantly increased expression of CD90 in hepatocellular carcinoma and cirrhosis. CD90 expression in different liver disease groups was evaluated by IHC staining score (from 0 to 12). We found the staining score was remarkably increased in HCC, compared with adjacent non-tumor tissues $(\mathrm{P}<0.0001)$ and normal liver tissues $(\mathrm{P}<0.001)$. But it shows no difference from cirrhosis or hepatitis samples. In addition, the staining score of cirrhosis was distinctly higher than those of adjacent non-tumor tissues $(\mathrm{P}<0.0001)$, hepatitis $(\mathrm{P}<0.01)$ and normal liver tissues $(\mathrm{P}<0.001)$. However, no differences were detected in other comparisons (Fig. 1C).

OCT4 was over expressed in hepatocellular carcinoma. These sections were also employed to determine expression of OCT4. None of normal liver tissues was detected with high level expression of OCT4 $(0 \%, 0 / 10)$. Furthermore, adjacent non-tumor, cirrhosis and hepatitis tissues all exhibited a relatively low rate of high expression of $34.9 \%$ (30/86), $27.3 \%$ $(6 / 22)$, and $36.4 \%(4 / 11)$, respectively. However, we found distinctly increased expression of OCT4 in HCC (60.5\%, 52/86)(Table 1, Fig. 2B). The subcellular location of OCT4 was predominantly expressed in the cytoplasm of cancer cells with moderate nuclear staining (Fig. 2A). The results of IHC score suggested that expression of OCT4 in HCC was evidently higher than the adjacent non-tumor $(\mathrm{P}<0.0001)$, cirrhosis $(\mathrm{P}<0.001)$ and normal liver tissues $(\mathrm{P}<0.01)$. Nevertheless, it showed no difference between HCC and hepatitis. Interestingly, we found increased expression of OCT4 in hepatitis compared to normal liver $(\mathrm{P}<0.05)$ (Fig. $2 \mathrm{C})$.
Correlation of CD90 and OCT4 expression with clinicopathologic parameters in HCC. We analyzed the relationships between the expression of these two proteins and clinicopathologic parameters, such as gender, age, HBsAg, AFP, cirrhosis, tumor size, pathological grade, tumor encapsulation, satellite lesion, PVTT and recurrence. As listed in Table 2, the expression of CD90 was positively associated with pathological grade $(\mathrm{P}=0.030)$, satellite lesion $(\mathrm{P}=0.029)$, PVTT $(\mathrm{P}=0.021)$ and recurrence $(\mathrm{P}=0.010)$. Moreover, OCT4 expression was positively correlated with tumor size $(\mathrm{P}=0.042)$, pathological grade $(\mathrm{P}=0.008)$, and recurrence $(\mathrm{P}<0.001)$, too (Table 2$)$.

Independent prognostic factors of HCC. In order to determine the risk factors related to postoperative Overall Survival (OS) and Disease free Survival (DFS), CD90, OCT4 and clinicopathologic parameters were firstly evaluated by univariate analysis. The factors with statistical significance were than adopted in the Cox regression model. Using the univariate analysis, we observed that risk factors for OS of HCC were CD90 expression, OCT4 expression, tumor size, pathological grade and PVTT. Similar results were found for DFS of HCC. The significant factors were CD90 expression, OCT4 expression, AFP, pathological grade and PVTT (all $\mathrm{P}<0.05$ ). The results of Cox regression multivariate analysis showed that CD90 expression, OCT4 expression, pathological grade and PVTT were risk factors both for OS of HCC and DFS of HCC (Table 3).

CD90 and OCT4 are both independent biomarkers for prognosis of HCC patients. According to CD90 expression level, HCC patients were divided into two groups: the high-expression group $(\mathrm{n}=58)$ and the low-expression group $(n=28)$. The results of Kaplan-Meier analysis showed that the 1-, 3- and 5- year OS rates were of low-expression CD90 group

Table 3. Prognostic factors for OS and DFS by univariate and multivariate analyses in HCC patients

\begin{tabular}{|c|c|c|c|c|c|c|}
\hline \multirow[t]{3}{*}{ Variables } & \multicolumn{3}{|c|}{ Overall Survival (OS) } & \multicolumn{3}{|c|}{ Disease-free Survival (DFS) } \\
\hline & \multirow[t]{2}{*}{ Univariate } & \multicolumn{2}{|c|}{ Multivariate } & \multirow[t]{2}{*}{ Univariate } & \multicolumn{2}{|c|}{ Multivariate } \\
\hline & & HR (95\% CI) & $\mathbf{P}$ & & HR (95\% CI) & $\mathbf{P}$ \\
\hline CD90 & $0.003^{*}$ & $2.096(1.076-4.084)$ & $0.030^{\star}$ & $0.004^{*}$ & $1.865(1.014-3.430)$ & $0.045^{\star}$ \\
\hline OCT4 & $<0.001^{*}$ & $2.341(1.248-4.393)$ & $0.008^{*}$ & $0.004^{*}$ & $1.914(1.086-3.371)$ & $0.025^{*}$ \\
\hline Gender male vs. female & 0.860 & - & NA & 0.381 & - & NA \\
\hline Age (years) $\geq 60$ vs. $<60$ & 0.326 & - & NA & 0.575 & - & NA \\
\hline HBsAg & 0.387 & - & NA & 0.259 & - & NA \\
\hline $\operatorname{AFP}(\mathrm{ng} / \mathrm{ml}) \geq 400$ vs. $<400$ & 0.068 & - & NA & $0.023^{*}$ & $0.996(0.553-1.794)$ & 0.989 \\
\hline Cirrhosis & 0.516 & - & NA & 0.541 & - & NA \\
\hline Tumor Size $(\mathrm{cm}) \geq 5$ vs. $<5$ & $0.047^{*}$ & $1.560(0.885-2.747)$ & 0.124 & 0.148 & - & NA \\
\hline Pathological grade (P: M: W) & $0.006^{*}$ & $0.608(0.378-0.977)$ & $0.040^{\star}$ & $0.010^{*}$ & $0.571(0.345-0.945)$ & $0.029^{*}$ \\
\hline Tumor encapsulation & 0.292 & - & NA & 0.512 & - & NA \\
\hline Satellite lesion & 0.178 & - & NA & 0.267 & - & NA \\
\hline PVTT & $0.009^{*}$ & $1.958(1.108-3.459)$ & $0.021^{*}$ & $0.001^{*}$ & $2.474(1.408-4.348)$ & $0.002^{*}$ \\
\hline
\end{tabular}

* Statistically significant $(\mathrm{P}<0.05)$

HBsAg, hepatitis B surface antigen; AFP, Alpha-fetoprotein; P, poorly differentiated; M, moderately differentiated; W, well differentiated; PVTT, portal vein tumor thrombi; 
was evidently higher than that of high-expression CD90 group $(\mathrm{P}=0.003)$ (Table 3, Fig. 3A). Furthermore, the 1-, 3- and 5year DFS rates of low-expression group were also remarkably higher than those of high-expression group $(\mathrm{P}=0.004)$ (Table 3, Fig. 3B). These results demonstrated that low-expression CD90 group obviously has better survival rates than the highexpression group.

In the same way, 86 HCC patients were divided into two groups according to the OCT4 expression level: the highexpression group $(n=52)$ and the low-expression group $(\mathrm{n}=34)$. The results were similar with CD90: compared with high-expression OCT4 group, the low-expression OCT4 group possessed distinctly higher OS rates $(\mathrm{P}<0.001)$ (Table 3, Fig. 3C). Meanwhile, the 1-, 3- and 5- year DFS rates of low-expression OCT4 group were significantly higher than high-expression OCT4 group $(\mathrm{P}=0.004)$ (Table 3, Fig. 3D).
Consequently, we could conclude that the high level of OCT4 expression predicts poor survival.

CD90 expression is not correlated with OCT4. The correlation between CD90 and OCT4 was then determined at their protein expression levels. We employed Spearman correlation analysis to determine the association between CD90 and OCT4 expression, considering them as two continuous variables. However, we failed to observe correlation $(r=0.145$, $\mathrm{P}=0.183$ ) (Fig. 4A). Next, the expression level of CD90 and OCT4 was counted as two categorical variables, and the frequency distribution of them was listed in contingency tables. The frequency distribution results also demonstrated no correlation between CD90 and OCT4 expression, as analyzed by Chi-square test ( $\mathrm{P}=0.974)$ (Fig. 4B).

Low expression of both CD90 and OCT4 is associated with favorable prognosis. To further analyze the prognostic

\section{CD90}
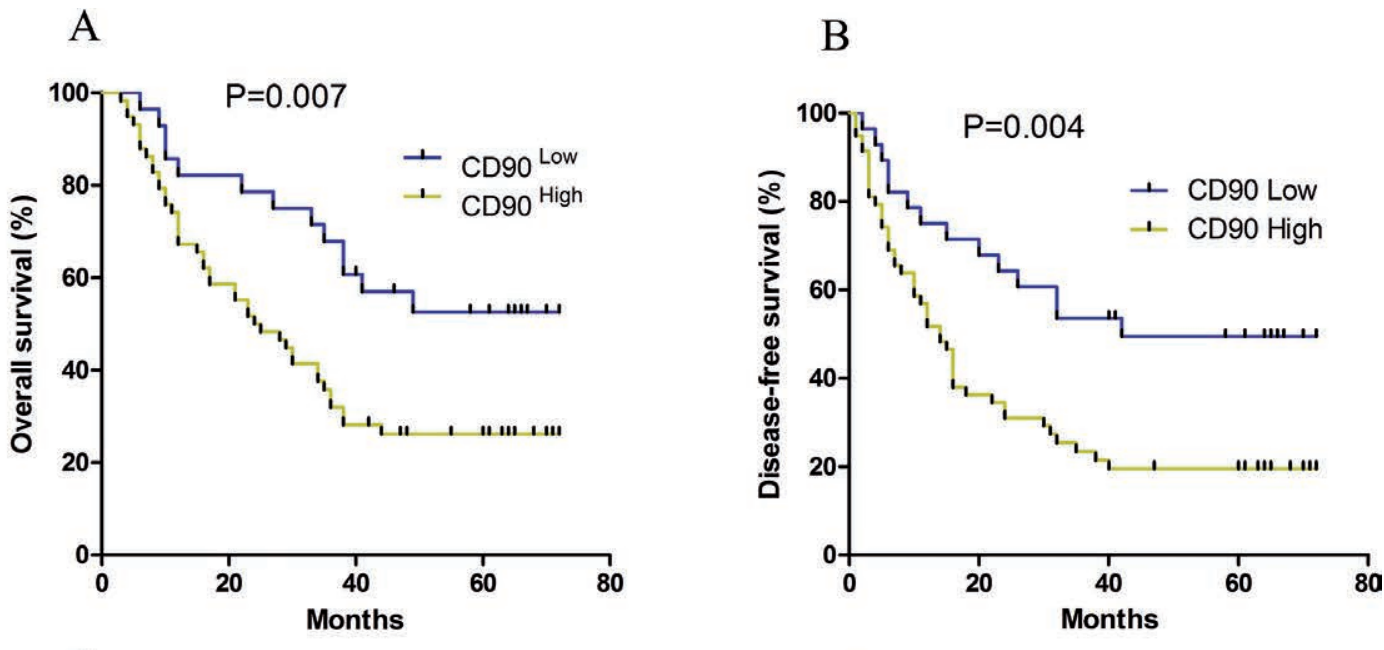

$\mathrm{C}$

D
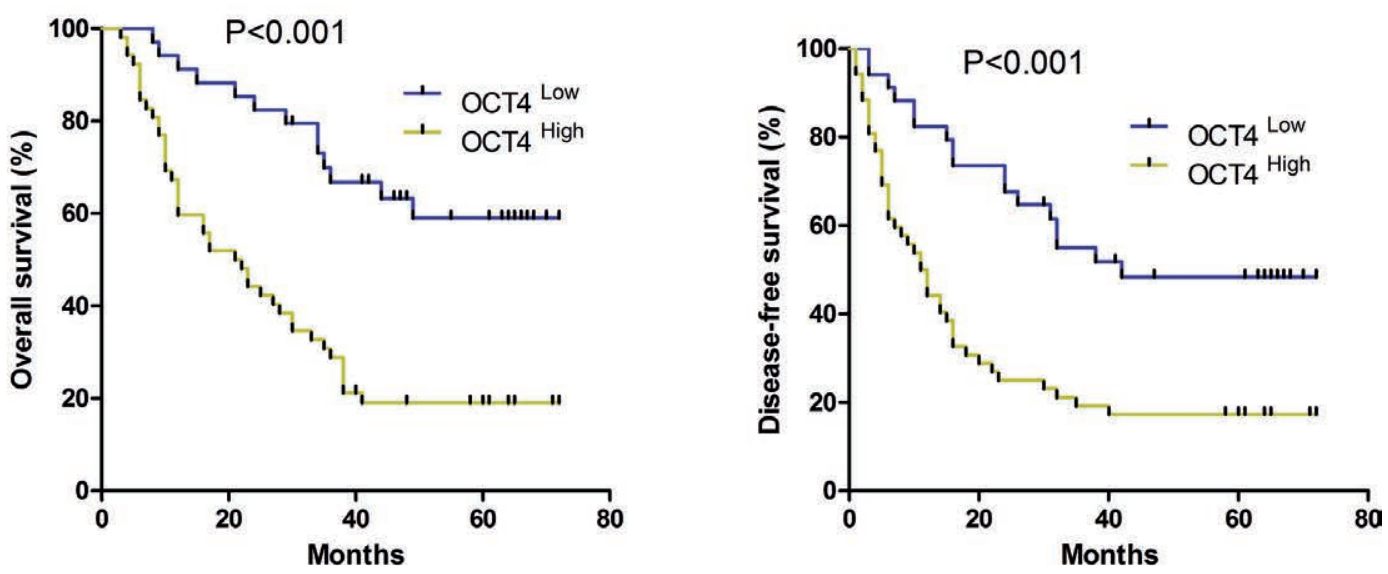

Figure 3. Kaplan-Meier survival curves of HCC patients after surgery for HCC according to CD90 and OCT4 expression. (A, B) Compared with CD90high expression group, the overall survival time and disease-free survival time were significantly higher in the $\mathrm{CD} 90$-low expression group. (C, D) Compared with OCT4-high expression group, the overall survival time and disease-free survival time were was significantly higher in the OCT4-low expression group. 
A

$\mathrm{r}=0.145, p=0.183$

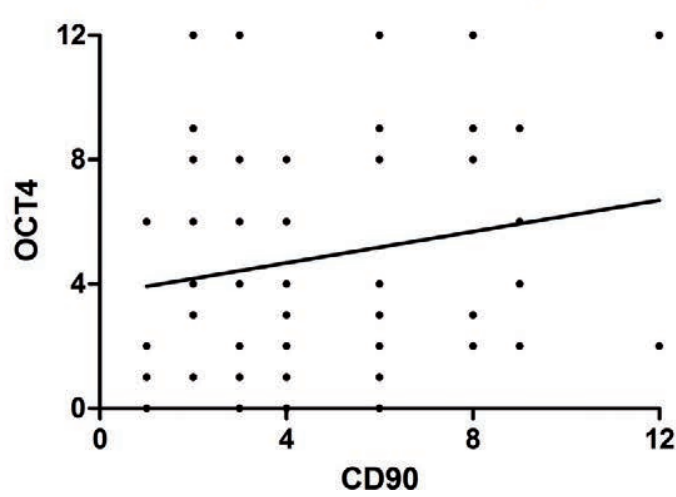

B

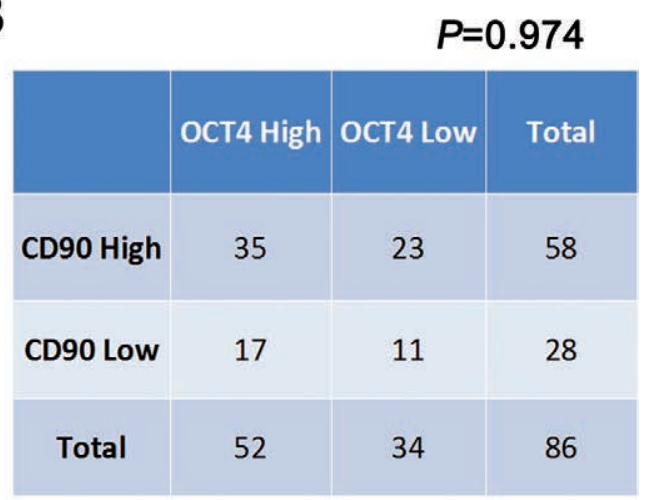

Figure 4. CD90 expression is not correlated with OCT4. (A) Spearman correlation analysis showed no association between CD90 and OCT4 expression. (B) Chi-square test demonstrated no correlation between CD90 and OCT4 expression when these two proteins were counted as categorical variables as well.

value of combining CD90 and OCT4 for HCC, we divided patients into four groups as below: CD90 ${ }^{\text {Low }} / \mathrm{OCT} 44^{\text {Low }}, \mathrm{CD} 90$ High /OCT4 Low, CD90 Low /OCT4 $4^{\text {High }}$ and CD90 High /OCT4 High. The survival curve demonstrated that CD90 Low /OCT4 Low group showed best survival rates for both OS and DFS. Other three subgroups showed remarkably decreased OS and RFS rates. Notably, the CD90 ${ }^{\text {High }}$ /OCT4 ${ }^{\text {High }}$ group had the worst clinical outcomes.

The 1-, 3- and 5- year OS rates of CD90 Low /OCT4 Low group were obviously higher than those of CD90 Low /OCT4 High group $(\mathrm{P}=0.012)$, and CD90 High $/ \mathrm{OCT} 44^{\text {High }}$ group $(\mathrm{P}<0.001)$. Similar results were observed for DFS rates. The 1-, 3- and 5year DFS rates of CD90 Low /OCT4 ${ }^{\text {Low }}$ group were remarkably higher than those of CD90 Low $/ \mathrm{OCT} 4{ }^{\text {High }}$ group $(\mathrm{P}=0.024)$ and CD90 High $/ O C T 44^{\text {High }}$ group $(\mathrm{P}<0.001)$ (Fig. 3$)$. Whereas it demonstrated no difference between CD90 Low /OCT4 ${ }^{\text {High }}$ group and CD90 High /OCT4 ${ }^{\text {Low }}$ group for OS $(\mathrm{P}=0.059)$ or DFS $(\mathrm{P}=0.051)$. These results propose that lower level of both CD90 and OCT4 predicts favorable prognosis. On the contrary, higher expression of both proteins is associated with the poorest clinical outcome (Fig. 5).

\section{Discussion}

In the present study, we investigated the variation of expression of CD90 and OCT4 in different types of liver diseases. We

$$
\begin{aligned}
& \text { 土 CD90 Low OCT4 }{ }^{\text {Low }}(\mathrm{n}=11) \\
& \text { 土 } \mathrm{CD}_{90}{ }^{\text {High }} \text { OCT4 }{ }^{\text {Low }}(\mathrm{n}=23) \\
& +\mathrm{CD}{ }^{\text {Low }} \text { OCT4 }{ }^{\text {High }}(\mathrm{n}=17) \\
& +\mathrm{CD} 90^{\text {High }} \text { OCT4 }{ }^{\mathrm{High}}(\mathrm{n}=35)
\end{aligned}
$$

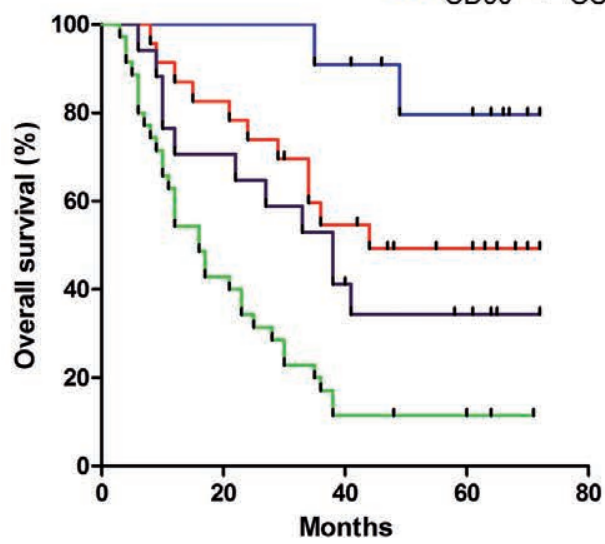

B
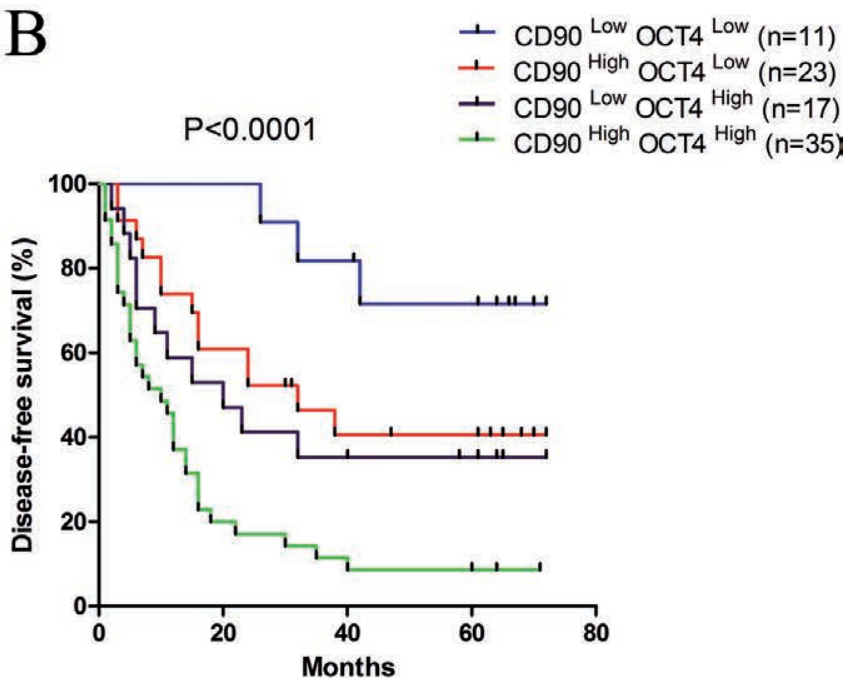

Figure 5. The combination of CD90 and OCT4 was found to enhance accuracy of predicting prognosis for HCC patients. (A) Overall survival curve. (B) Disease-free survival curve. 
found that CD90 was mainly expressed in CAFs. Expression levels of CD90 and OCT4 in HCC were remarkably higher than in adjacent non-tumor and normal liver tissues. KaplanMeier analysis demonstrated HCC patients with a higher level of CD90 or OCT4 suffered a shorter post-operation survival than the low-expression groups. Furthermore, patients with both high expression of these two markers had the worst prognosis.

CD90 has been considered as a candidate biomarker for CSCs in diverse malignancies, such as HCC [28], esophageal cancer [29], and glioma [30]. Fan et al isolated $\mathrm{CD} 90^{+} \mathrm{CD} 45$ cells from HCC tissues and found it had stronger tumorigenic capacity, compared with CD90-CD45 subsets. Furthermore, the $\mathrm{CD} 90^{+} \mathrm{CD} 45^{-}$subpopulation was detected in more than $90 \%$ of blood samples and all of tumor specimens of HCC patients [28]. But there still existed different voices. Another research demonstrated that no expression of CD90 was detected in the hepatic oval/progenitor cell population. Instead, they reported CD90 as a maker of satellite cells/myofibroblasts subpopulation [31]. Sukowati et al [13] found that CAFs isolated from human HCC tissues were positive for CD90. In addition, Chen et al' study [15] identified CD90 as one of marker of $\mathrm{CAFs}$ and demonstrated that $\mathrm{CD} 90^{+} \mathrm{CAFs}$ promoted stem-like properties in lung cancer cells. Evidences from multiple studies indicated CD90 as a candidate marker of CAFs [13-15, 32]. Our findings showed that most of CD90 was mainly expressed in CAFs and some endothelium, which were consistent with previous findings in pancreatic carcinoma [32], lung cancer [15] and HCC [14]. Like previous studies [13, 28], our results demonstrated that CD90 was up-regulated in HCC tissues. Interestingly, not HCC, but cirrhosis samples showed highest expression of CD90. The reason may be that cirrhosis tissues contains abundant fibroblasts which are the origin of CD90. To further determine the prognostic value of CD90, we analyzed relationships between $\mathrm{CD} 90$ and clinicopathologic parameters. We also found positive correlation between CD90 and several clinicopathologic parameters: pathological grade, satellite lesion and PVTT, which was consistent with the concept that CD90 promoted metastatic properties of tumors $[12,29]$. We divided HCC patients into two groups according to CD90 expression and discovered that it could be an independent predictor for prognosis. So we concluded that $\mathrm{CD} 0^{+}$tumor stroma cells have an impact on clinical outcome of HCC patients, which had not been reported before.

Although a similar study about OCT4 was performed by Dong et al. [26], they only detected OCT4 expression in HCC and cirrhosis samples. As it is a sequential progress from hepatitis, cirrhosis to HCC $[33,34]$, we additionally examined OCT4 expression in adjacent non-tumor, hepatitis and normal liver sections, conferring a comprehensive recognition of OCT4 expression in the progression of HCC. Immunohistochemical staining revealed higher expression of OCT4 in HCC, compared to adjacent non-tumor, cirrhosis, and normal liver sections, indicating a significant up-regulation of OCT4 protein in HCC tissues. However, we failed to find difference between HCC and hepatitis sections. This may be due to that inflammatory reaction activated the self-renewal property of hepatic cells with reactive increase of OCT4. Besides, relatively small sample size with only 11 hepatitis specimens may limit detection of statistical differences. Approximately consistent with previous study [26], our data showed OCT4 expression positively correlated with tumor size, pathological grade and recurrence. The results of Kaplan-Meier analysis exhibited that HCC patients with high level of OCT4 had a poorer postoperation survival.

Yang et al used $\mathrm{CD} 90^{+} \mathrm{CAFs}$ as feeder cells to co-culture with lung cancer cells and generated OCT4 ${ }^{+} \mathrm{CSC}$. Moreover, they observed that CAFs secreted IGF-II for paracrine regulation on cancer cells [15]. It is therefore of interest to investigate the correlation of CD90 and OCT4 in HCC patients. However, we failed to find correlation between these two proteins by neither chi-square test nor linear regression. This may be explained as followings: Firstly, there existed methodology unicity that we only adopted immunohistochemical staining method and limited examination to protein level. Testing expression at transcription level may have higher efficiency of detecting correlation. Secondly, discoveries in lung cancer may be not suitable for HCC duo to heterogeneity between different types of cancers. CSCs of HCC may be not influence by CAFs as much as lung cancer because of existence of IGFII autocrine regulation in HCC [35], which was not observed in lung cancer.

The function of tumor associated stroma cells on the initiation and progression of malignancies had always been ignored, before the theory of tumor microenvironment (TME) was proposed and extensively accepted in recent years. As it said, tumor associated stroma cells interacted with tumor cells and influenced disease initiation, progression and patient prognosis together [7]. Hence, combination of tumor stroma cells maker CD90 and CSCs marker OCT4 may yield more information and enhance the accuracy of prediction for prognosis of HCC patients. Our results suggested that their combination confers a better prognostic value, compared with either one alone. For instance, HCC patients with both high CD90 and high OCT4 expression had the poorest OS and DFS rates, while those patients with both low expression had the most favorable survival. The second best prognosis belonged to these patients with high CD90 expression and low OCT4 expression, implying OCT4 may be a more important factor for prognosis of patient with HCC than CD90. After all, the CSCs is the true endopathic cause and the tumor associated stroma cells are just promptive factors.

In conclusion, we demonstrate that expression of CD90 and OCT4 are increased in HCC and higher expression of these two proteins is correlated with poor prognosis of HCC patients. Although no relationship between CD90 and OCT4, patients with double high expression of CD90 and OCT4 had shortest OS and DFS. Furthermore, we found that the predictive range of CD90 combined with OCT4 expression was more sensitive than ether CD90 or OCT4 alone. From 
a diagnostic viewpoint, our study indicated that combined detection of CD90 and OCT4 could be employed as a new marker to predict prognosis of HCC patients.

Acknowledgments: This research was supported by "The National Natural Science Foundation of China (grant no. 81172372). We thank Qun-Ying Li, Xiu-Qun Li and Jian-Guo Qi for technical support.

\section{References}

[1] JEMAL A, SIEGEL R, XU J, WARD E. Cancer statistics, 2010. CA Cancer J Clin. 2010; 60: 277-300. http://dx.doi. org/10.3322/caac.20073

[2] AUTRET A, MARTIN SJ. Bcl-2 family proteins and mitochondrial fission/fusion dynamics. Cell Mol Life Sci. 2010; 67: 1599-606. http://dx.doi.org/10.1007/s00018-010-0286-x

[3] EL-SERAG HB. CURRENT CONCEPTS Hepatocellular Carcinoma. New England Journal Of Medicine. 2011; 365: 1118-27. http://dx.doi.org/10.1056/NEJMra1001683

[4] FLOREANI A, FARINATI F. Risk factors associated with hepatocellular carcinoma in primary biliary cirrhosis. Hepatology. 2013; 58: 1520-1. http://dx.doi.org/10.1002/ $\underline{\text { hep. } 26355}$

[5] MITTAL S, EL-SERAG HB. Epidemiology of hepatocellular carcinoma: consider the population. Journal of clinical gastroenterology. 2013; 47 Suppl: S2-6. http://dx.doi.org/10.1097/ MCG.0b013e3182872f29

[6] FAN ST, LO CM, POON RTP, YEUNG C, LIU CL, et al. Continuous Improvement of Survival Outcomes of Resection of Hepatocellular Carcinoma A 20-Year Experience. Annals of surgery. 2011; 253: 745-58. http://dx.doi.org/10.1097/ $\underline{\text { SLA.0b013e3182111195 }}$

[7] JOYCE JA, POLLARD JW. Microenvironmental regulation of metastasis. Nature reviews Cancer. 2009; 9: 239-52. http:// dx.doi.org/10.1038/nrc2618

[8] FUKUMURA D, XAVIER R, SUGIURA T, CHEN Y, PARK EC, et al. Tumor induction of VEGF promoter activity in stromal cells. Cell. 1998; 94: 715-25. http://dx.doi.org/10.1016/ $\underline{\text { S0092-8674(00)81731-6 }}$

[9] SAALBACH A, WETZIG T, HAUSTEIN UF, ANDEREGG U. Detection of human soluble Thy-1 in serum by ELISA - Fibroblasts and activated endothelial cells are a possible source of soluble Thy-1 in serum. Cell Tissue Res. 1999; 298(2): 307-15. http://dx.doi.org/10.1007/s004419900079

[10] CRAIG W, KAY R, CUTLER RL, LANSDORP PM. Expression Of Thy-1 on Human Hematopoietic Progenitor Cells. J Exp Med. 1993; 177: 1331-42. http://dx.doi.org/10.1084/ jem.177.5.1331

[11] SAMANIEGO R, ESTECHA A, RELLOSO M, LONGO N, ESCAT JL, et al. Mesenchymal Contribution to Recruitment, Infiltration, and Positioning of Leukocytes in Human Melanoma Tissues. J Invest Dermatol. 2013; 133: 2255-64. http://dx.doi.org/10.1038/jid.2013.88

[12] SCHUBERT K, GUTKNECHT D, KOBERLE M, ANDEREGG U, SAALBACH A. Melanoma Cells Use Thy-1 (CD90) on Endothelial Cells for Metastasis Formation. Am
J Pathol. 2013; 182: 266-76. http://dx.doi.org/10.1016/j. ajpath.2012.10.003

[13] SUKOWATI CH, ANFUSO B, TORRE G, FRANCALANCI P, CROCE LS, et al. The expression of CD90/Thy-1 in hepatocellular carcinoma: an in vivo and in vitro study. PloS one. 2013; 8: e76830. http://dx.doi.org/10.1371/journal. pone. 0076830

[14] SUKOWATI CH, ANFUSO B, CROCE LS, TIRIBELLI C. The role of multipotent cancer associated fibroblasts in hepatocarcinogenesis. BMC cancer. 2015; 15: 188. http://dx.doi. org/10.1186/s12885-015-1196-y

[15] CHEN WJ, HO CC, CHANG YL, CHEN HY, LIN CA, et al. Cancer-associated fibroblasts regulate the plasticity of lung cancer stemness via paracrine signalling. Nature communications. 2014; 5: 3472. http://dx.doi.org/10.1038/ $\underline{\text { ncomms } 4472}$

[16] NICHOLS J, ZEVNIK B, ANASTASSIADIS K, NIWA H, KLEWE-NEBENIUS D, et al. Formation of pluripotent stem cells in the mammalian embryo depends on the POU transcription factor Oct4. Cell. 1998; 95: 379-91. http://dx.doi. org/10.1016/S0092-8674(00)81769-9

[17] TAKAHASHI K, YAMANAKA S. Induction of pluripotent stem cells from mouse embryonic and adult fibroblast cultures by defined factors. Cell. 2006; 126: 663-76. http://dx.doi. org/10.1016/j.cell.2006.07.024

[18] HAY DC, SUTHERLAND L, CLARK J, BURDON T. Oct-4 knockdown induces similar patterns of endoderm and trophoblast differentiation markers in human and mouse embryonic stem cells. Stem cells. 2004; 22: 225-35. http:// dx.doi.org/10.1634/stemcells.22-2-225

[19] BOYER LA, LEE TI, COLE MF, JOHNSTONE SE, LEVINE SS, et al. Core transcriptional regulatory circuitry in human embryonic stem cells. Cell. 2005; 122: 947-56. http://dx.doi. org/10.1016/j.cell.2005.08.020

[20] EZEH UI, TUREK PJ, REIJO RA, CLARK AT. Human embryonic stem cell genes OCT4, NANOG, STELLAR, and GDF3 are expressed in both seminoma and breast carcinoma. Cancer. 2005; 104: 2255-65. http://dx.doi.org/10.1002/cncr.21432

[21] ATLASI Y, MOWLA SJ, ZIAEE SA, BAHRAMI AR. OCT-4, an embryonic stem cell marker, is highly expressed in bladder cancer. International journal of cancer Journal international du cancer. 2007; 120: 1598-602. http://dx.doi.org/10.1002/ ijc. 22508

[22] LIU CG, LU Y, WANG BB, ZHANG YJ, ZHANG RS, et al. Clinical implications of stem cell gene Oct-4 expression in breast cancer. Annals of surgery. 2011; 253: 1165-71. http:// dx.doi.org/10.1097/SLA.0b013e318214c54e

[23] GE N, LIN HX, XIAO XS, GUO L, XU HM, et al. Prognostic significance of Oct 4 and Sox2 expression in hypopharyngeal squamous cell carcinoma. Journal of translational medicine. 2010; 8: 94. http://dx.doi.org/10.1186/1479-5876-8-94

[24] CHENG L, SUNG MT, COSSU-ROCCA P, JONES TD, MACLENNAN GT, et al. OCT4: biological functions and clinical applications as a marker of germ cell neoplasia. The Journal of pathology. 2007; 211: 1-9. http://dx.doi.org/10.1002/ path. 2105 
[25] GIDEKEL S, PIZOV G, BERGMAN Y, PIKARSKY E. Oct$3 / 4$ is a dose-dependent oncogenic fate determinant. Cancer cell. 2003; 4: 361-70. http://dx.doi.org/10.1016/S1535-6108(03)00270-8

[26] DONG Z, ZENG Q, LUO H, ZOU J, CAO C, et al. Increased expression of OCT4 is associated with low differentiation and tumor recurrence in human hepatocellular carcinoma. Pathology, research and practice. 2012; 208: 527-33. http:// dx.doi.org/10.1016/j.prp.2012.05.019

[27] GION M, MIONE R, LEON AE, LUFTNER D, MOLINA R, et al. CA27.29: a valuable marker for breast cancer management. A confirmatory multicentric study on 603 cases. European journal of cancer. 2001; 37: 355-63. http://dx.doi.org/10.1016/ $\underline{\text { S0959-8049(00)00396-8 }}$

[28] YANG ZF, HO DW, NG MN, LAU CK, YU WC, et al. Significance of CD90+ cancer stem cells in human liver cancer. Cancer cell. 2008; 13: 153-66. http://dx.doi.org/10.1016/j. ccr.2008.01.013

[29] TANG KH, DAI YD, TONG M, CHAN YP, KWAN PS, et al. A CD90(+) tumor-initiating cell population with an aggressive signature and metastatic capacity in esophageal cancer. Cancer research. 2013; 73: 2322-32. http://dx.doi.org/10.1158/00085472.CAN-12-2991

[30] HE J, LIU Y, ZHU T, ZHU J, DIMECO F, et al. CD90 is identified as a candidate marker for cancer stem cells in primary high-grade gliomas using tissue microarrays. Molecular \& cellular proteomics : MCP. 2012; 11: M111 010744.

[31] DEZSO K, JELNES P, LASZLO V, BAGHY K, BODOR C, et al. Thy- 1 is expressed in hepatic myofibroblasts and not oval cells in stem cell-mediated liver regeneration. Am J Pathol. 2007; 171: 1529-37. http://dx.doi.org/10.2353/ajpath.2007.070273

[32] ZHU J, THAKOLWIBOON S, LIU X, ZHANG M, LUBMAN DM. Overexpression of CD90 (Thy-1) in pancreatic adenocarcinoma present in the tumor microenvironment. PloS one. 2014;9: e115507. http://dx.doi.org/10.1371/journal. pone. 0115507

[33] PAN N, CHEN K, QIU J, SUN H, XU J, et al. Human leukocyte antigen class I alleles and haplotypes associated with primary hepatocellular carcinoma in persistent $\mathrm{HBV}$-infected patients. Human immunology. 2013; 74: 758-63. http://dx.doi. org/10.1016/j.humimm.2013.02.007

[34] MERICAN I, GUAN R, AMARAPUKA D, ALEXANDER MJ, CHUTAPUTTI A, et al. Chronic hepatitis B virus infection in Asian countries. Journal of gastroenterology and hepatology. 2000; 15: 1356-61. http://dx.doi.org/10.1046/j.1440-1746 $.2000 .0150121356 . \mathrm{x}$

[35] SHAN J, SHEN J, LIU L, XIA F, XU C, et al. Nanog regulates self-renewal of cancer stem cells through the insulin-like growth factor pathway in human hepatocellular carcinoma. Hepatology. 2012; 56: 1004-14. http://dx.doi.org/10.1002/hep.25745 